\title{
Selected CSF biomarkers indicate no evidence of early neuroinflammation in Huntington disease
}

\section{OPEN}

Tua Vinther-Jensen, MD, PhD*

Lars Börnsen, MD, PhD*

Esben Budtz-Jørgensen,

$\mathrm{PhD}$

Cecilie Ammitzbøll, MD

Ida U. Larsen, MSc

Lena E. Hjermind, MD, $\mathrm{PhD}$

Finn Sellebjerg, MD,

DMSc, PhD

Jørgen E. Nielsen, MD, $\mathrm{PhD} \ddagger$

Correspondence to

Dr. Nielsen:

jnielsen@sund.ku.dk

\section{ABSTRACT}

Objective: To investigate CSF biomarkers of neuroinflammation and neurodegeneration in Huntington disease (HD) gene-expansion carriers compared to controls and to investigate these biomarkers in association with clinical HD rating scales and disease burden score.

Methods: We collected CSF from 32 premanifest and 48 manifest HD gene-expansion carriers and 24 gene-expansion negative at-risk controls. We examined biomarkers of neuroinflammation (matrix metalloproteinase 9, C-X-C motif chemokine 13, terminal complement complex, chitinase-3-like-protein 1 [CHI3L1], and osteopontin [OPN]) and neurodegeneration (microtubuleassociated protein tau, neurofilament light polypeptide [NFL], and myelin basic protein [MBP]). The study was approved by the Ethics Committee of the Capital Region of Denmark $(\mathrm{H} 2-$ 2011-085) and written informed consent was obtained from each participant before enrollment.

Results: NFL was the only biomarker that increased in premanifest stages and no evidence of early involvement of neuroinflammation in HD was found. However, we found that the biomarkers for neurodegeneration, MBP and tau, increased during the disease course in manifest HD geneexpansion carriers and were associated with an increase of the neuroinflammation biomarkers CHI3L1 and OPN. Tau was also increased in all gene-expansion carriers with psychiatric symptoms compared to gene-expansion carriers without psychiatric symptoms.

Conclusions: Neuroinflammation, which seems not to be an early event in our cohort, may be secondary to neurodegeneration in late HD. NFL is a possible disease burden correlate in HD, reflecting neuronal loss even before motor symptom onset, and may be useful as a dynamic biomarker in intervention studies. Neurol Neuroimmunol Neuroinflamm 2016;3:e287; doi: 10.1212/NXI.0000000000000287

\section{GLOSSARY}

CAP = CAG age product; CHI3L1 = human chitinase 3-like 1 protein; CRP $=$ C-reactive protein; CXCL13 = C-X-C motif chemokine 13; HD = Huntington disease; IgG = immunoglobulin G; MBP = myelin basic protein; MMP9 = matrix metalloproteinase-9; NFL = neurofilament light polypeptide; NOx = nitric oxide and nitrite/nitrate; OPN = osteopontin; OR = odds ratio; $\mathbf{T a u}=$ microtubule-associated protein tau (total); TFC = total functional capacity; TMS = total motor score; UHDRS = Unified Huntington's Disease Rating Scale.

Huntington disease (HD) is an autosomal dominant progressive neurodegenerative disorder caused by an expanded CAG repeat in the huntingtin gene. ${ }^{1} \mathrm{HD}$ is characterized by involuntary movements, psychiatric disturbances, and cognitive decline. ${ }^{2}$ Despite intensive research, the pathogenesis of HD remains unknown; however, the mutated huntingtin protein results in neuronal dysfunction and eventually neuronal death. Several pathophysiologic mechanisms have been suggested, including a neuroinflammatory component. ${ }^{3}$

Multiple studies conducted on postmortem HD brain tissue and mouse models of HD have found altered expression of immunologically active molecules in the CNS. ${ }^{4-7}$ One study reported

\footnotetext{
*These authors contributed equally to this work.

$¥$ These authors contributed equally to this work.

From the Neurogenetics Clinic, Danish Dementia Research Centre (T.-V.J., I.U.L., L.E.H., J.E.N.), and Danish Multiple Sclerosis Center (L.B., C.A., F.S.), Department of Neurology, Rigshospitalet, Department of Cellular and Molecular Medicine, Section of Neurogenetics (T.-V.J., L.E.H., J.E.N.), Section of Biostatistics (E.B.-J.), and Department of Psychology (I.U.L.), University of Copenhagen, Denmark.

Funding information and disclosures are provided at the end of the article. Go to Neurology.org/nn for full disclosure forms. The Article Processing charge was paid by the authors.

This is an open access article distributed under the terms of the Creative Commons Attribution-NonCommercial-NoDerivatives License 4.0 (CC BY-NC-ND), which permits downloading and sharing the work provided it is properly cited. The work cannot be changed in any way or used commercially.
} 
increased levels of clusterin in CSF from manifest gene-expansion carriers, ${ }^{8}$ and imaging studies using the PK11195 ligand indicated increased microglial activity in manifest and premanifest HD gene-expansion carriers. ${ }^{9,10}$ The huntingtin gene is ubiquitously expressed, and the pathologic changes are not limited to the CNS as systemic abnormalities, including an altered peripheral immune profile, are also described. Biomarkers of inflammation have been shown to be increased in plasma from HD gene-expansion carriers and the upregulation has been observed up to 16 years before predicted onset, ${ }^{4,5,8}$ although in a recent study these findings were not confirmed. ${ }^{11}$

In this study, we measured biomarkers in 104 CSF samples, drawn and handled under standardized conditions, from HD gene-expansion carriers and HD gene-expansion negative controls. No evidence for early neuroinflammation was found. We found, however, that biomarkers of neurodegeneration increased in manifest disease, which was also associated with an increase in biomarkers of neuroinflammation.

METHODS HD gene-expansion carriers and controls. CSF samples from 3 groups were analyzed: premanifest geneexpansion carriers, manifest gene-expansion carriers, and geneexpansion negative individuals. Participants' recruitment, exclusion and inclusion criteria, and investigation program is reported elsewhere. ${ }^{12}$ Participants were classified according to the Unified Huntington's Disease Rating Scale (UHDRS) total motor score (TMS) ${ }^{13}$ with a UHDRS-TMS of $>5$ defined as motor manifest HD gene-expansion carriers. If the score was $\leq 5$, indicating no substantial motor signs, they were classified as premanifest HD gene-expansion carriers. Participants were categorized based on the evaluation of psychiatric symptoms and performance on cognitive testing as described in VintherJensen et al. ${ }^{14}$ Disease burden was calculated as CAG age product $(\mathrm{CAP})$ score: $\mathrm{CAP}=\left(\left[\mathrm{CAG}_{\mathrm{n}}-33.66\right] \times\right.$ age $) .{ }^{15}$

The study was approved by the Ethics Committee of The Capital Region of Denmark (H2-2011-085) and written informed consent was obtained from each participant before enrollment.

CSF studies. CSF samples were obtained by lumbar puncture and processed as previously described. ${ }^{12}$ Blood samples were drawn for corresponding blood biochemistry including leukocyte count and C-reactive protein (CRP) to control for possible infections. The following ELISAs were used to measure CSF biomarkers: C-X-C motif chemokine 13 (CXCL13), Quantikine ELISA Kit (R\&D Systems, Minneapolis, MN); matrix metalloproteinase-9 (MMP9), Quantikine ELISA Kit; total nitric oxide and nitrite/nitrate $\left(\mathrm{NO}_{\mathrm{x}}\right)$ assay; human osteopontin (OPN) and human chitinase 3-like 1 protein (CHI3L1), Quantikine ELISA Kit; neurofilament light polypeptide (NFL), ELISA (UmanDiagnostics, Umeå, Sweden); myelin basic protein (MBP) ELISA (Beckman Coulter, Sharon Hill, PA); TCC ELISA Kit (Hycult Biotech, Uden, Netherlands); and microtubule-associated protein tau (total) (Tau) human ELISA Kit (Life Technologies, Nærum, Denmark). ELISAs were performed according to the manufacturers' instructions. Samples were measured in duplicate and the mean intra-assay variability was $22.4 \%$ for CXCL13, $7.7 \%$ for MMP9, $5.5 \%$ for $\mathrm{NO}_{\mathrm{X}}$, $3.2 \%$ for OPN, $4.7 \%$ for CHI3L1, $3.5 \%$ for NFL, $4.9 \%$ for MBP, $5.5 \%$ for TCC, and $2.9 \%$ for Tau.

Statistical analysis. Data are shown as mean and SD or as median and range. Biomarkers were corrected for skewed levels with logistic function prior to analysis. All measured concentrations of the values were adjusted for age and sex; regression coefficients $b$ and $p$ values are annotated. Adjustments for age and sex were calculated from a regression analysis that included age, sex, and group as covariates. The adjustments were based on an assumption that the age effect was the same in the 3 groups; this assumption was tested in a regression analysis with an interaction between age and group. Comparisons of concentration levels between the groups were performed using linear regression with adjustment for age and sex, corrected for multiple comparisons with Bonferroni correction. Determination of the individual biomarkers' association with traditional disease progression scales and cumulative CAG toxicity expressed as CAP score was tested with linear regression analysis including age, sex in all geneexpansion carriers, and UHDRS total functional capacity $(\mathrm{TFC})^{16}$ and UHDRS-TMS scores in manifest gene-expansion carriers. Correlations and partial correlations (after CAP score adjustment) between the individual biomarkers were performed using Pearson $r$, while $p$ values were Bonferroni-corrected. The levels of the biomarkers in relation to gene-expansion carriers with psychiatric symptoms or cognitive impairment were tested using multiple regression analysis including the adjusted values of the biomarkers as the dependent variable, and CAP score and a dichotomous variable indicating symptom group as independent variables. To test that the effect of the CAP score was the same in the 2 groups, a regression analysis with an interaction between CAP score and symptom group was applied.

RESULTS CSF samples were drawn from 121 individuals, 104 of whom were included in the analysis. The remaining samples from 3 gene-expansion negative controls and 8 premanifest and 6 manifest geneexpansion carriers were excluded due to high erythrocyte count $(>500$ cells $/ \mathrm{mL})$. The included participants were $24 \mathrm{HD}$ gene-expansion negative controls and 32 premanifest and 48 manifest geneexpansion carriers. Participant characteristics are shown in table 1 and further described in earlier publications from our group. ${ }^{12,14}$ Immunoglobulin G (IgG) index was not significantly different in the 3 groups ( $p=$ 0.224 ) and the distribution of individuals who had an IgG index above normal $(>0.67)$ did not differ in the 3 groups ( $p=0.294$ ) either. CSF oligoclonal bands were detected in 3 gene-expansion negative controls, in 3 manifest HD gene-expansion carriers, and in 9 premanifest gene-expansion carriers. CSF leukocyte counts were not significantly different in the 3 groups ( $p=0.392$, Kruskal-Wallis). All participants had normal blood leukocyte count and CRP.

Age and sex effects on the CSF biomarkers. NFL and MBP were associated with both age and sex (expressed 


\begin{tabular}{|c|c|c|c|c|c|c|c|c|}
\hline \multirow[t]{2}{*}{ Table 1} & dat & & & & & & & \\
\hline & No. & $\mathrm{M} / \mathrm{F}$ & Age, y & CAG repeat length & CAP $^{a}$ score & Albumin ratiob & IgG index & Oligoclonal bands ${ }^{c}$ \\
\hline Manifest gene-expansion carriers & 48 & $29 / 19$ & $50(30-74)$ & $43(40-53)$ & $468.0(101.8)$ & $5.45(2.36)$ & $0.54(0.09)$ & 3 \\
\hline Premanifest gene-expansion carriers & 32 & $19 / 13$ & $36(20-54)$ & $43(39-48)$ & 319.3 (99.3) & $5.05(2.56)$ & $0.58(0.22)$ & 9 \\
\hline HD gene-expansion negative & 24 & $13 / 11$ & $42(26-68)$ & $18(17-26)$ & NR & $4.92(1.43)$ & $0.53(0.23)$ & 3 \\
\hline
\end{tabular}

Abbreviations: $\mathrm{CAP}=\mathrm{CAG}$ age product; $\mathrm{HD}=$ Huntington disease; IgG = immunoglobulin $\mathrm{G}$ index; $\mathrm{NR}=$ not relevant.

Data are presented as mean (SD) or median (range).

${ }^{a}$ CAP score $=\left(\left[\mathrm{CAG}_{n}-33.66\right] \times\right.$ age $)$.

${ }^{\mathrm{b}}$ Albumin ratio $\times 10^{-3}=\left(\mathrm{CSF}_{\text {albumin }} / \mathrm{P}_{\text {albumin }}\right) \times 1,000$.

${ }^{\mathrm{c}}$ Number of participants with oligoclonal bands.

as male compared to female participants) ( $b=0.009$, $p<0.0001$, age and $13.2 \%$ higher levels in male participants, $p=0.030$, for NFL; and $b=0.006$, $p<0.001$, age and $8.1 \%$ higher levels in male participants, $p=0.004$, for MBP). Associated with age but not sex were Tau $(b=0.003, p=0.027$, age and $8.8 \%$ higher levels in male participants, $p=0.135)$; CHI3L1 $(b=0.007, p<0.0001$, age and $8.8 \%$ higher levels in male participants, $p=0.104)$; TCC ( $b=0.011, p=$ 0.001 , age and $8.6 \%$ higher levels in male participants, $p=0.385) ; \mathrm{NO}_{\mathrm{x}}(b=0.003, p=0.001$, age and $9.2 \%$ higher levels in male participants, $p=0.073$ ); and OPN $(b=0.006, p<0.0001$, age and $9.2 \%$ higher levels in male participants, $p=0.165$ ).

Concentration differences between the groups. Figure 1 shows the adjusted levels for the 7 biomarkers in the 3 groups.

Subsequently, interaction analyses between age and group were performed to investigate if the age effect differed significantly among the 3 groups. Only Tau showed different effects of age in the 3 groups ( $p<$ 0.001 ) and was dependent on age solely in manifest gene-expansion carriers. As a result, Tau was only adjusted for sex and not age in subsequent analyses.

MMP9 was only detected in 3 samples (all with increased IgG index and oligoclonal bands), while CXCL13 was detected in 7 (3 with increased IgG index and 4 with oligoclonal bands). Neither the pattern of detection ( $p=0.914$ [MMP)], $p=0.938$ [CXCL13]) nor the risk, expressed as odds ratio (OR), of having a detectable sample differed significantly in the 3 groups (OR $0.670, p=0.786$ for MMP9; OR $0.818, p=0.838$ for CXCL13) when comparing gene-expansion negative controls with manifest gene-expansion carriers and when comparing gene-expansion negative controls with premanifest gene-expansion carriers (OR 0.962, $p=0.962$ for MMP9; OR 0.703, $p=0.529$ for CXCL13).

Relationship between biomarkers and disease progression scales. Analyses of the relationship of age- and sexadjusted biomarkers to disease progression scales were subsequently performed. In manifest gene- expansion carriers, $\mathrm{CHI} 3 \mathrm{~L} 1, \mathrm{OPN}, \mathrm{TCC}$, and $\mathrm{NO}_{\mathrm{x}}$ were not associated with UHDRS-TFC or UHDRSTMS. For both NFL $(p=0.065)$ and MBP $(p=$ 0.078 ), there was a trend towards association with the UHDRS-TFC scores. Furthermore, NFL but not MBP was associated with UHDRS-TMS ( $p=$ 0.022). Tau was not associated with UHDRS-TFC, but a trend towards association was found for UHDRS-TMS $(p=0.075)$. In all gene-expansion carriers, CAP scores were significantly associated with MBP $(p=0.001)$, CHI3L1 $(p=0.010)$, Tau $(p=0.01)$, and NFL $(p<0.0001)$ (figure 2$)$.

Correlation between biomarkers. In all gene-expansion carriers, the correlation and the partial correlation of the adjusted concentration of the biomarkers showed a strong association between NFL and MBP $(R=0.509, p<0.0001)$; however, the association was lost when controlling for CAP score $(R=0.301, p=0.073)$ (table 2). Correlations between the adjusted levels of the biomarkers were repeated in the gene-expansion negative controls. The correlations that were significant were the same in the 2 analyses, apart from the correlations between OPN and Tau $(R=0.517, p=0.073)$ and OPN and CHI3L1 $(R=0.443, p=0.23)$ (data not shown).

Correlation between HD symptoms and biomarkers. Only Tau and TCC were associated with psychiatric symptoms. Tau showed different effects of the CAP score in the 2 groups, with or without psychiatric symptoms $(p=0.005)$. Thus, Tau only increased as a function of CAP score in HD gene-expansion carriers without psychiatric symptoms but was at a constantly higher level in HD gene-expansion carriers with psychiatric symptoms. TCC levels in HD gene-expansion carriers with psychiatric symptoms were higher than TCC levels in HD gene-expansion carriers without psychiatric symptoms $(p=0.015)$. NFL was the only biomarker associated with cognitive impairment, with HD gene-expansion carriers with cognitive impairment having higher levels of NFL than cognitively intact $\mathrm{HD}$ gene-expansion carriers $(p=$ 0.012). 
A

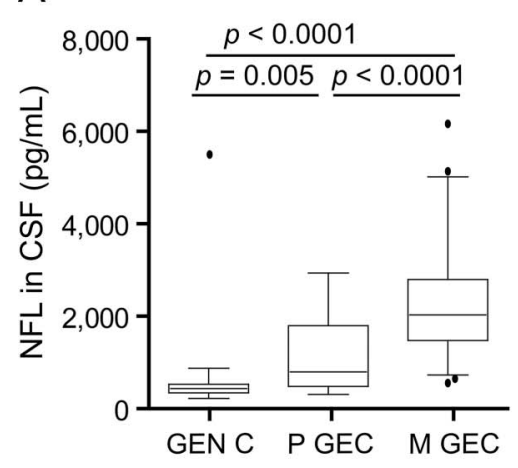

D

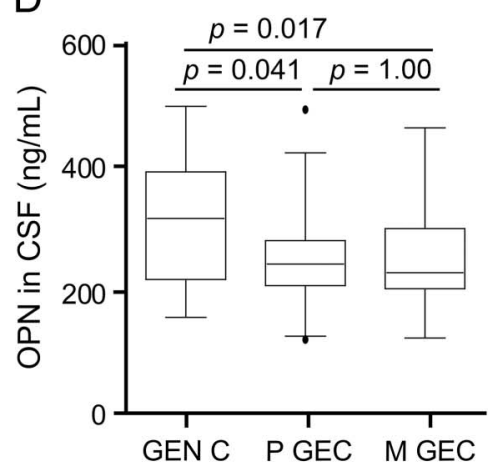

G

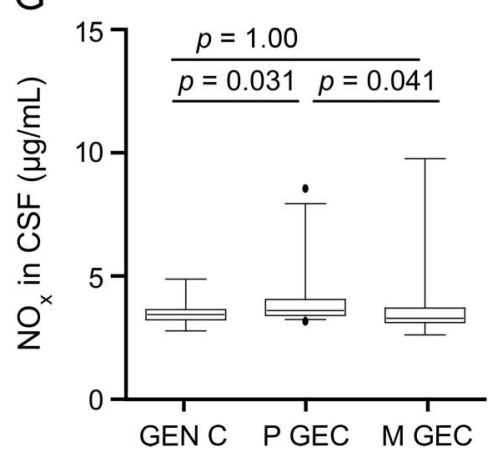

B

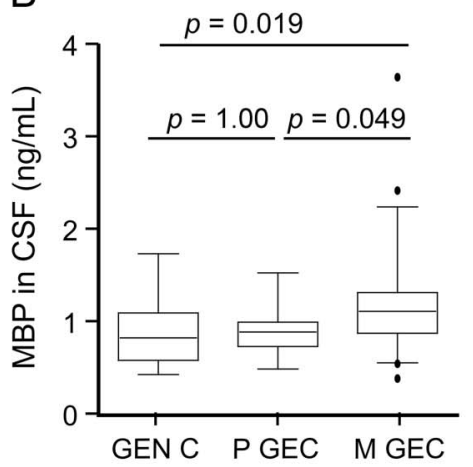

E

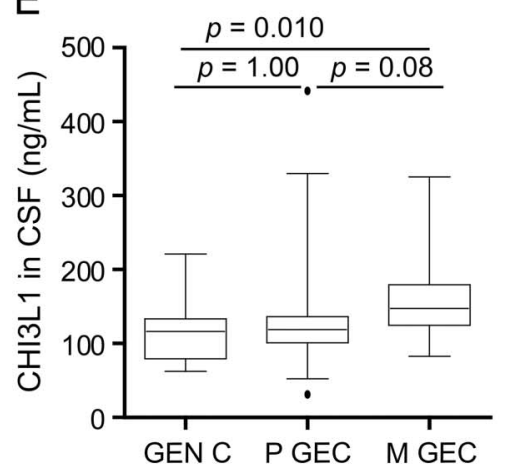

C

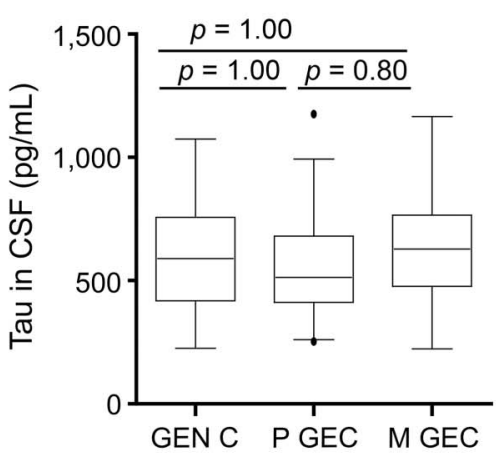

F

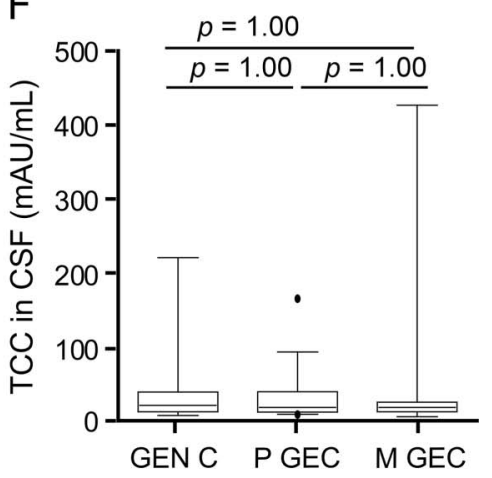

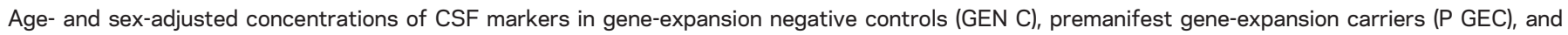

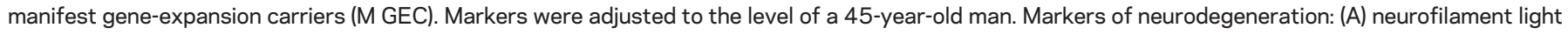

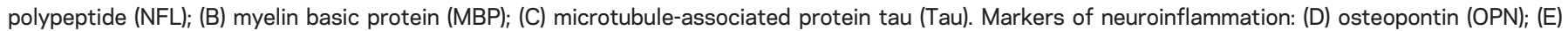

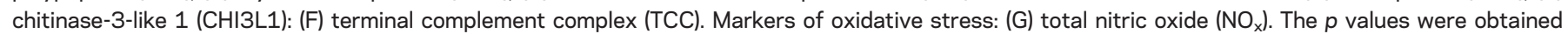
from linear regression analysis and Bonferroni correction.

DISCUSSION In this controlled study of CSF inflammation in HD, we examined a total of 104 CSF samples from 32 premanifest and 48 manifest $\mathrm{HD}$ gene-expansion carriers and 24 gene-expansion negative at-risk controls. All samples were drawn, processed, and stored according to a standardized procedure. We examined several biomarkers of neuroinflammation and neurodegeneration and their correlation with motor, psychiatric, and cognitive measures. We found no evidence of early inflammatory involvement; however, we found evidence of sequential evolvement of biomarkers of neurodegeneration and neuroinflammation. The examined biomarkers fell into 2 categories. One category primarily consisted of specific inflammatory biomarkers (MMP), CXCL13, TCC, and $\left.\mathrm{NO}_{\mathrm{x}}\right)$. These biomarkers were not increased in manifest gene-expansion carriers and showed no correlation with disease burden or other biomarkers. The second category comprised biomarkers of neurodegeneration (NFL, MBP, and Tau) and the biomarker of inflammation CHI3L1. These biomarkers were increased in manifest HD gene-expansion carriers, correlated to disease burden, and showed intercorrelation with the other biomarkers in this category. OPN did not fit in either 
A

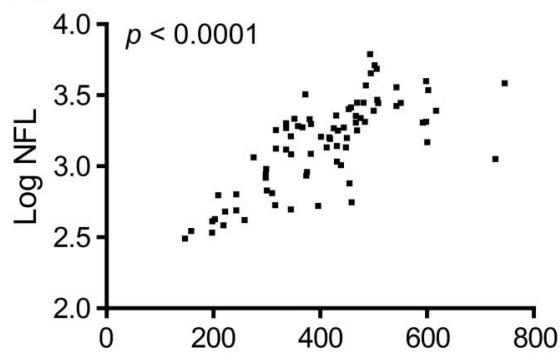

C

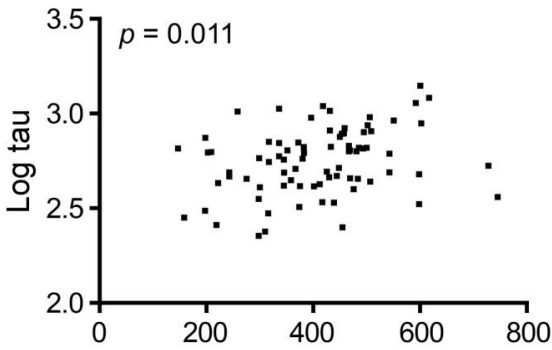

$\mathrm{E}$

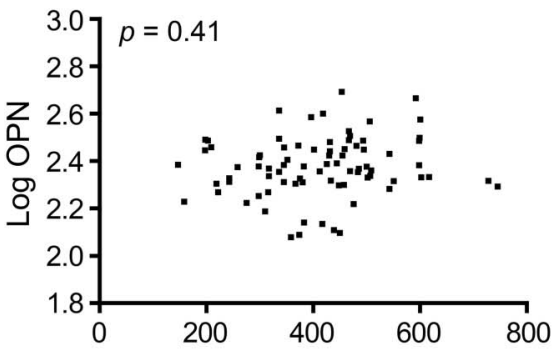

G

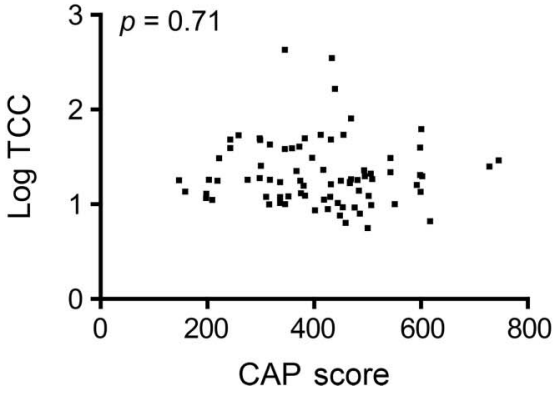

B

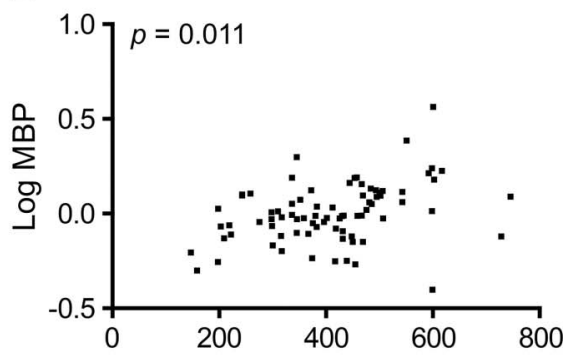

$\mathrm{D}$

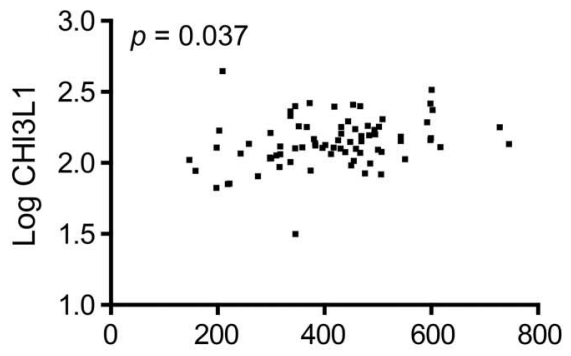

$\mathrm{F}$

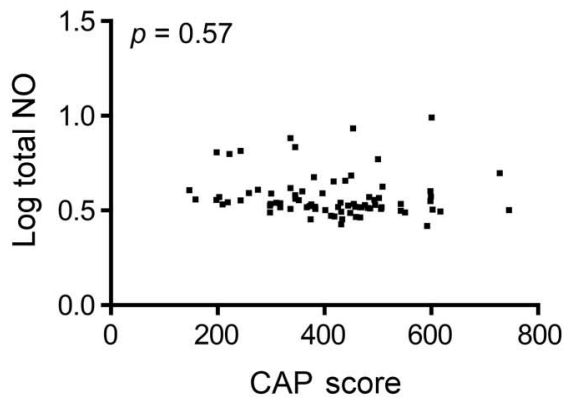

Sex- and age-adjusted log-transformed concentrations of the CSF markers as a function of CAP score ([CAG $\mathrm{n}^{-33.66] \times}$ age). (A) Neurofilament light polypeptide (NFL); (B) myelin basic protein (MBP); (C) microtubule-associated protein tau (Tau) (only sex-adjusted); (D) chitinase-3 like 1 (CHI3L1); (E) osteopontin (OPN); (F) total nitric oxide $\left(\mathrm{NO}_{x}\right)$; (G) terminal complement complex (TCC). The $p$ values were obtained from linear regression analyses including sex, age, and CAP score.

group as it was marginally decreased in HD geneexpansion carriers and did not correlate with disease burden but was closely correlated with the biomarkers in the second category and therefore subsequently included there.

MMP9 and CXCL13 are considered to be biomarkers of leukocyte trafficking into the subarachnoid and perivascular space during neuroinflammation. MMP9 activity has been linked to increased blood-brain barrier permeability ${ }^{17}$ and CXCL13 is a B-cell chemoattractant. ${ }^{18}$ Previous studies have found increased expression levels of MMP9 in postmortem HD brain tissue and increased levels of MMP9 in plasma samples from patients with HD and transgenic HD mice.,19 Our data do not indicate a blood-brain barrier dysfunction as CSF leukocyte counts, and the albumin quotient did not differ in the 3 groups. However, CSF oligoclonal bands were found in 9 premanifest 


\begin{tabular}{|c|c|c|c|c|c|}
\hline \multirow[t]{2}{*}{ Table 2} & \multicolumn{5}{|c|}{ Intercorrelation and partial intercorrelation of the adjusted markers } \\
\hline & NFL & MBP & $\mathrm{Tau}^{\mathrm{a}}$ & CHI3L1 & OPN \\
\hline MBP & $0.509^{b}$ & & & & \\
\hline Tau & $0.388^{c}$ & $0.514^{d}$ & & & \\
\hline CHI3L1 & $0.330^{c}$ & $0.389^{e}$ & $0.471^{b}$ & & \\
\hline OPN & 0.11 & $0.317^{c}$ & $0.510^{\mathrm{b}}$ & $0.393^{\mathrm{e}}$ & \\
\hline $\mathrm{NO}_{x}$ & -0.192 & 0.117 & 0.046 & 0.034 & 0.033 \\
\hline \multicolumn{6}{|c|}{$\begin{array}{l}\text { Partial correlations, controlled } \\
\text { for CAP score }\end{array}$} \\
\hline MBP & 0.301 & & & & \\
\hline Tau & 0.227 & $0.452^{d}$ & & & \\
\hline CHI3L1 & 0.168 & 0.327 & $0.416^{e}$ & & \\
\hline OPN & 0.049 & 0.374 & $0.518^{b}$ & $0.390^{\mathrm{e}}$ & \\
\hline $\mathrm{NO}_{x}$ & -0.155 & 0.217 & 0.086 & 0.073 & 0.037 \\
\hline
\end{tabular}

Abbreviations: CHI3L1 = chitinase-3-like 1; MBP = myelin basic protein; NFL = neurofilament light polypeptide; OPN = osteopontin; $\mathrm{NO}_{\mathrm{x}}=$ total nitric oxide; $\mathrm{Tau}=$ microtubuleassociated protein tau (total).

Analyzed by Pearson correlation, $R$. The $p$ values are one-sided, Bonferroni corrected.

${ }^{\text {a }}$ Only sex-adjusted.

${ }^{b} p<0.0001$.

${ }^{\mathrm{c}} \mathrm{p}<0.05$.

${ }^{d} p<0.001$

e $p<0.01$.

${ }^{f} \mathrm{CAG}$ age product (CAP). The directions of all the correlation were characterized by increases in both biomarkers and CAP score.

gene-expansion carriers, which may indicate the presence of an aspect of neuroinflammation not detected by our assays that may be present in the premanifest stage.

The complement pathway is a powerful humoral component of the innate immune system..$^{20}$ Increased levels of TCC have been measured in several studies where the complement pathway is suspected to be activated. ${ }^{21}$ A previous study showed an increased expression of complement activators and regulators in HD brain tissue, suggesting an activation of the complement pathway. ${ }^{7}$ We did not find increased TCC in HD gene-expansion carriers compared to gene-expansion negative controls, which could indicate that (1) there is no complement pathway activation, (2) the activation may be present locally on the cell surfaces in the striatum and thus not reaching the CSF, or (3) the increased expression of complement activators is limited to early activation of the complement cascade but not terminal activation with generation of TCC.

Oxidative stress has been linked to the activation of microglia, ${ }^{22}$ biomarkers of inflammation in other neuroinflammatory conditions, ${ }^{23}$ and is suspected to have a major role in the neuronal damage and degeneration in $\mathrm{HD} .{ }^{24}$ We found $\mathrm{NO}_{\mathrm{x}}$ to be elevated in premanifest but not in manifest HD gene-expansion carriers compared to gene-expansion negative controls and with no correlation to disease burden in either premanifest gene-expansion carriers alone (data not shown) or all HD gene-expansion carriers. This finding implies that there is increased NO synthase activity in the preclinical stages of HD, but the pathophysiologic relevance of this finding is unclear.

NFL is an unspecific but sensitive biomarker of neuronal and axonal damage in neurodegenerative disorders. ${ }^{25}$ Two previous HD CSF studies have shown elevated NFL levels in CSF from HD geneexpansion carriers. The first study used CSF from 35 manifest HD gene-expansion carriers and correlated increased NFL to UHDRS-TMS but not with CAG repeat length. ${ }^{26} \mathrm{~A}$ recent study from Wild et al. ${ }^{27}$ found that NFL was associated with the level of mutant huntingtin but not to cognitive function when controlling for disease burden. In our larger independent cohort, we confirm significant correlations of NFL with both UHDRS-TMS and CAG repeat length as included in CAP score. In contrast to another study of CSF markers in $\mathrm{HD},{ }^{27}$ we found that $\mathrm{HD}$ gene-expansion carriers with cognitive impairment had higher CSF levels of NFL, as compared to cognitively intact HD gene-expansion carriers controlling for disease burden (CAP score), possibly indicating a more severe degree of neurodegeneration in cognitively impaired HD geneexpansion carriers. This discrepancy may arise from the fact that this study only included 12 participants in the analysis of NFL and cognitive function. ${ }^{27}$

NFL was also the only biomarker that was elevated in both premanifest and manifest HD geneexpansion carriers compared to controls. These results suggest that NFL potentially reflects the neurodegeneration in HD from an early time point, long before onset of motor symptoms. NFL correlated with MBP and $\mathrm{CHI} 3 \mathrm{~L} 1$, but these correlations disappeared when controlling for disease burden, reflecting that NFL may be a biomarker of disease burden.

Tau and MBP concentrations are elevated in a variety of neurodegenerative disorders as measures of different aspects of neurodegeneration, myelin breakdown, and neuronal and axonal damage. ${ }^{28,29}$ In contrast to NFL, Tau and MBP appear to reflect later stages of the neurodegeneration in $\mathrm{HD}$, since Tau and MBP concentrations were only increased and correlated with the disease burden in manifest gene-expansion carriers but not in premanifest geneexpansion carriers. Two earlier studies have investigated Tau in CSF and reported increased levels in manifest gene-expansion carriers; however, Tau was not correlated to any disease scores. ${ }^{27,30}$ In our study the levels of Tau did, however, differ in HD geneexpansion carriers with or without psychiatric symptoms. HD gene-expansion carriers with psychiatric symptoms had elevated levels of Tau in general, whereas in HD gene-expansion carriers without 
Figure 3 Hypothesized model of the dynamics of CSF biomarkers in Huntington disease (HD)

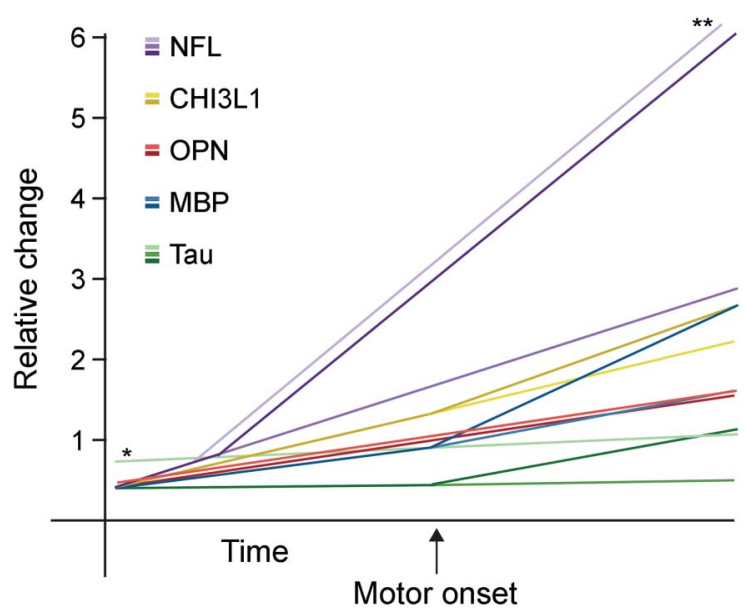

The dynamics of CSF biomarkers over time are illustrated for gene-expansion negative controls (medium-toned lines) and the changes from gene-expansion negative controls to geneexpansion carriers are illustrated by dark-toned lines. Light-toned lines: * illustrates level of microtubule-associated protein tau (total) (Tau) in HD gene-expansion carriers with psychiatric symptoms; ** illustrates level of neurofilament light polypeptide in HD gene-expansion carriers with cognitive impairment. $\mathrm{CHI} 3 \mathrm{~L} 1$ = human chitinase 3 -like 1 protein; $\mathrm{MBP}=$ myelin basic protein; NFL = neurofilament light polypeptide; OPN = osteopontin.

psychiatric symptoms, Tau increased with disease burden. Earlier studies on Tau and neuropsychiatric disorders are sparse, include low numbers of patients, and have yielded conflicting results. ${ }^{31}$ Our results may reflect that HD gene-expansion carriers with psychiatric symptoms already have neuronal and axonal damage prior to motor onset.

While OPN is involved in various pathologic and physiologic processes such as bone mineralization, wound repair, and immunity, ${ }^{32}$ CHI3L1, a chitinbinding protein lacking enzymatic activity, is known to play a role in cancer and chronic inflammatory disease. ${ }^{33}$ We chose OPN and CHI3L1 as biomarkers of CNS inflammation, since their CSF concentrations are increased in various inflammatory diseases of the CNS, e.g., multiple sclerosis, but also in other CNS diseases primarily considered as being neurodegenerative disorders but with accumulating evidence of an inflammatory component, e.g., Alzheimer disease. ${ }^{34,35}$ In the present study, we found increased CHI3L1 levels in manifest but not in premanifest geneexpansion carriers, confirming the results of our previous study of CHI3L1 ${ }^{36}$ This may indicate that there is an inflammatory component in manifest $\mathrm{HD}$ even though OPN levels were not increased. As seen in early multiple sclerosis, ${ }^{37}$ we found a positive correlation between OPN and CHI3L1 and Tau. In controls, $\mathrm{OPN}$ and $\mathrm{CHI} 3 \mathrm{~L} 1$ concentrations are higher in the CSF than in the plasma, ${ }^{34}$ suggesting that OPN and CHI3L1 are synthesized intrathecally under physiologic conditions, independent of ongoing inflammation. In the CNS, OPN and CHI3L1 are expressed by microglia and astrocytes, respectively. ${ }^{38,39}$ These cells are hypothesized to be involved in HD pathogenesis. ${ }^{6,10,40}$ However, we cannot exclude that noninflammatory processes may alter the expression of OPN or CHI3L1 in astrocytes, microglia, or other CNS cells in HD and that the increased level of CHI3L1 is not an indication of neuroinflammation per se. Indeed, OPN and CHI3L1 may play physiologic roles in tissue regeneration and repair. OPN was decreased in the premanifest group but not the manifest group, which might indicate an early but not late malfunction of microglia. More markers of microglia/ macrophage function should be measured to investigate this further. There are several isoforms of OPN and the different commercial ELISA kits do not take this into account; hence the results of OPN measurements should be interpreted with caution. ${ }^{41}$

Figure 3 presents a hypothesized model on the dynamics of the investigated CSF biomarkers.

We found that NFL may be a relevant correlate of disease burden in all HD gene-expansion carriers reflecting neuronal loss even before motor symptom onset, and may be useful as a dynamic biomarker in future intervention studies.

Although our cohort is relatively large, our study is exploratory and needs validation, ideally also including a positive control group as, e.g., multiple sclerosis. Furthermore, measurements of the relevant markers in plasma or serum are needed to investigate a possible relation between CSF and blood levels of the markers. Despite those limitations, our study does not provide evidence of neuroinflammation in early HD pathogenesis and the increased CSF concentrations of CHI3L1 in manifest HD gene-expansion carriers are not substantiated by other inflammatory biomarkers and may reflect other pathologic processes in HD. However, the fact that 9 presymptomatic gene-expansion carriers did have oligoclonal bands deserves attention in future studies and additional cross-sectional and longitudinal studies on more CSF biomarkers of inflammation and neurodegeneration are necessary to fully clarify the contribution of neuroinflammation in HD pathogenesis.

\section{AUTHOR CONTRIBUTIONS}

T. Vinther-Jensen: research project conception, organization, and execution; statistical analysis design, execution, review, and critique; writing of the first draft; and manuscript review and critique. L. Börnsen: research project conception and execution, writing of the first draft, and manuscript review and critique. E. Budtz-Jørgensen: statistical analysis design, review, and critique; and manuscript review and critique. C. Ammitzbøll: research project execution and manuscript review and critique. I.U. Larsen: research project organization and execution and manuscript review and critique. L.E. Hjermind: research project conception and manuscript review and critique. F. Sellebjerg: research project conception and organization, statistical analysis review and critique, and manuscript review and critique. J.E. Nielsen: research project conception and organization, statistical analysis review and critique, and manuscript review and critique. 


\section{STUDY FUNDING}

This project was supported by the Danish Huntington's Disease Association Research Foundation, the Aase and Ejnar Danielsen Foundation, the Danish Multiple Sclerosis Society, and the Novo Nordisk Foundation.

\section{DISCLOSURES}

T. Vinther-Jensen reports no disclosures. L. Börnsen received travel funding from Novartis and Genzyme and received research support from the Danish Multiple Sclerosis Society. E. Budtz-Jørgensen is an editorial advisory member for Environmental Health. C. Ammitzbøll received travel support from Genzyme. I.U. Larsen received research support from the University of Copenhagen. L.E. Hjermind reports no disclosures. F. Sellebjerg served on the scientific advisory board for Biogen Idec, Genzyme, Merck Serono, Sanofi-Aventis, Teva, and Novo Nordisk; received travel funding and/or speaker honoraria from Bayer Schering, Biogen Idec, Genzyme, Merck Serono, Novartis, Sanofi-Aventis, Schering-Plough, and Teva; is a section editor for Multiple Sclerosis and Related Disorders; consulted for Biogen Idec; and received research support from Biogen Idec, Sanofi-Aventis, Novartis, Danish Strategic Research Council, Danish Multiple Sclerosis Society, and Lounkær Foundation. J.E. Nielsen reports no disclosures. Go to Neurology. org/nn for full disclosure forms.

Received January 15, 2016. Accepted in final form August 22, 2016.

\section{REFERENCES}

1. The Huntington's Disease Collaborative Research Group. A novel gene containing a trinucleotide repeat that is expanded and unstable on Huntington's disease chromosomes. Cell 1993;72:971-983.

2. Roos RA. Huntington's disease: a clinical review. Orphanet J Rare Dis 2010;5:40.

3. Moller T. Neuroinflammation in Huntington's disease. J Neural Transm 2010;117:1001-1008.

4. Bjorkqvist M, Wild EJ, Thiele J, et al. A novel pathogenic pathway of immune activation detectable before clinical onset in Huntington's disease. J Exp Med 2008;205: 1869-1877.

5. Chang KH, Wu YR, Chen YC, Chen CM. Plasma inflammatory biomarkers for Huntington's disease patients and mouse model. Brain Behav Immun 2015;44:121-127.

6. Crotti A, Benner C, Kerman BE, et al. Mutant Huntingtin promotes autonomous microglia activation via myeloid lineage-determining factors. Nat Neurosci 2014;17: 513-521.

7. Singhrao SK, Neal JW, Morgan BP, Gasque P. Increased complement biosynthesis by microglia and complement activation on neurons in Huntington's disease. Exp Neurol 1999;159:362-376.

8. Dalrymple A, Wild EJ, Joubert R, et al. Proteomic profiling of plasma in Huntington's disease reveals neuroinflammatory activation and biomarker candidates. J Proteome Res 2007;6:2833-2840.

9. Politis M, Pavese N, Tai YF, et al. Microglial activation in regions related to cognitive function predicts disease onset in Huntington's disease: a multimodal imaging study. Hum Brain Mapp 2011;32:258-270.

10. Tai YF, Pavese N, Gerhard A, et al. Microglial activation in presymptomatic Huntington's disease gene carriers. Brain 2007;130:1759-1766.

11. Silajdzic E, Rezeli M, Vegvari A, et al. A critical evaluation of inflammatory markers in Huntington's disease plasma. J Huntington Dis 2013;2:125-134.

12. Vinther-Jensen $\mathrm{T}$, Simonsen AH, Budtz-Jorgensen E, Hjermind LE, Nielsen JE. Ubiquitin: a potential cerebrospinal fluid progression marker in Huntington's disease. Eur J Neurol 2015;22:1378-1384.
13. Huntington Study Group. Unified Huntington's Disease Rating Scale: reliability and consistency. Mov Disord 1996;11:136-142.

14. Vinther-Jensen T, Larsen IU, Hjermind LE, et al. A clinical classification acknowledging neuropsychiatric and cognitive impairment in Huntington's disease. Orphanet J Rare Dis 2014;9:114.

15. Zhang Y, Long JD, Mills JA, Warner JH, Lu W, Paulsen JS. Indexing disease progression at study entry with individuals at risk for Huntington disease. Am J Med Genet B Neuropsychiatr Genet 2011;156B:751-763.

16. Shoulson I, Fahn S. Huntington disease: clinical care and evaluation. Neurology 1979;29:1-3.

17. Ram M, Sherer Y, Shoenfeld Y. Matrix metalloproteinase9 and autoimmune diseases. J Clin Immunol 2006;26: 299-307.

18. Walker CA, Huttner AJ, O'Connor KC. Cortical injury in multiple sclerosis: the role of the immune system. BMC Neurol 2011;11:152.

19. Silvestroni A, Faull RL, Strand AD, Moller T. Distinct neuroinflammatory profile in post-mortem human Huntington's disease. Neuroreport 2009;20:1098-1103.

20. Rus H, Cudrici C, Niculescu F. The role of the complement system in innate immunity. Immunol Res 2005;33:103-112.

21. Sellebjerg F, Jaliashvili I, Christiansen M, Garred P. Intrathecal activation of the complement system and disability in multiple sclerosis. J Neurol Sci 1998;157:168-174.

22. Hanisch UK. Functional diversity of microglia: how heterogeneous are they to begin with? Front Cell Neurosci 2013;7:65.

23. Romme Christensen J, Bornsen L, Khademi M, et al. CSF inflammation and axonal damage are increased and correlate in progressive multiple sclerosis. Mult Scler 2013;19: 877-884.

24. Browne SE, Beal MF. Oxidative damage in Huntington's disease pathogenesis. Antioxid Redox Signal 2006;8:20612073.

25. Norgren N, Sundstrom P, Svenningsson A, Rosengren L, Stigbrand T, Gunnarsson M. Neurofilament and glial fibrillary acidic protein in multiple sclerosis. Neurology 2004;63:1586-1590.

26. Constantinescu R, Romer M, Oakes D, Rosengren L, Kieburtz K. Levels of the light subunit of neurofilament triplet protein in cerebrospinal fluid in Huntington's disease. Parkinsonism Relat Disord 2009;15:245-248.

27. Wild EJ, Boggio R, Langbehn D, et al. Quantification of mutant huntingtin protein in cerebrospinal fluid from Huntington's disease patients. J Clin Invest 2015;125: 1979-1986.

28. Blennow K, Zetterberg H, Fagan AM. Fluid biomarkers in Alzheimer disease. Cold Spring Harb Perspect Med 2012; 2:a006221.

29. Lamers KJ, Vos P, Verbeek MM, Rosmalen F, van Geel WJ, van Engelen BG. Protein S-100B, neuronspecific enolase (NSE), myelin basic protein (MBP) and glial fibrillary acidic protein (GFAP) in cerebrospinal fluid (CSF) and blood of neurological patients. Brain Res Bull 2003;61:261-264.

30. Constantinescu R, Romer M, Zetterberg H, Rosengren L, Kieburtz K. Increased levels of total tau protein in the cerebrospinal fluid in Huntington's disease. Parkinsonism Relat Disord 2011;17:714-715.

31. Rozenstein-Tsalkovich L, Kahana E, Shenker A, Baitcher F, Cohen OS, Kahana-Merhavi S. CSF tau 
protein in Alzheimer's disease and other neurological and psychiatric diseases. Austin Alzheimers J Parkinsons Dis 2014;1:10.

32. Wang KX, Denhardt DT. Osteopontin: role in immune regulation and stress responses. Cytokine Growth Factor Rev 2008;19:333-345.

33. Lee CG, Da Silva CA, Dela Cruz CS, et al. Role of chitin and chitinase/chitinase-like proteins in inflammation, tissue remodeling, and injury. Annu Rev Physiol 2011;73: 479-501.

34. Bornsen L, Khademi M, Olsson T, Sorensen PS, Sellebjerg F. Osteopontin concentrations are increased in cerebrospinal fluid during attacks of multiple sclerosis. Mult Scler 2011; $17: 32-42$.

35. Chowdhury SA, Lin J, Sadiq SA. Specificity and correlation with disease activity of cerebrospinal fluid osteopontin levels in patients with multiple sclerosis. Arch Neurol 2008;65:232-235.

36. Vinther-Jensen T, Budtz-Jorgensen E, Simonsen AH, Nielsen JE, Hjermind LE. YKL-40 in cerebrospinal fluid in Huntington's disease: a role in pathology or a nonspecific response to inflammation? Parkinsonism Relat Disord 2014;20:1301-1303.

37. Modvig S, Degn M, Horwitz $\mathrm{H}$, et al. Relationship between cerebrospinal fluid biomarkers for inflammation, demyelination and neurodegeneration in acute optic neuritis. PLoS One 2013;8:e77163.

38. Bonneh-Barkay D, Bissel SJ, Kofler J, Starkey A, Wang G, Wiley CA. Astrocyte and macrophage regulation of YKL40 expression and cellular response in neuroinflammation. Brain Pathol 2012;22:530-546.

39. Morimoto J, Kon S, Matsui Y, Uede T. Osteopontin as a target molecule for the treatment of inflammatory diseases. Curr Drug Targets 2010;11:494-505.

40. Sapp E, Kegel KB, Aronin N, et al. Early and progressive accumulation of reactive microglia in the Huntington disease brain. J Neuropathol Exp Neurol 2001;60:161-172.

41. Gimba ER, Tilli TM. Human osteopontin splicing isoforms: known roles, potential clinical applications and activated signaling pathways. Cancer Lett 2013;331:11-17. 


\section{Neurology \\ Neuroimmunology \& Neuroinflammation}

\section{Selected CSF biomarkers indicate no evidence of early neuroinflammation in Huntington disease}

Tua Vinther-Jensen, Lars Börnsen, Esben Budtz-Jørgensen, et al.

Neurol Neuroimmunol Neuroinflamm 2016;3;

DOI 10.1212/NXI.0000000000000287

This information is current as of September 28, 2016

Updated Information \&

Services

References

Citations

Subspecialty Collections

Permissions \& Licensing

Reprints including high resolution figures, can be found at:

http://nn.neurology.org/content/3/6/e287.full.html

This article cites 41 articles, 2 of which you can access for free at: http://nn.neurology.org/content/3/6/e287.full.html\#\#ref-list-1

This article has been cited by 3 HighWire-hosted articles: http://nn.neurology.org/content/3/6/e287.full.html\#\#otherarticles

This article, along with others on similar topics, appears in the following collection(s):

Huntington's disease

http://nn.neurology.org//cgi/collection/huntingtons_disease

Information about reproducing this article in parts (figures,tables) or in its entirety can be found online at:

http://nn.neurology.org/misc/about.xhtml\#permissions

Information about ordering reprints can be found online:

http://nn.neurology.org/misc/addir.xhtml\#reprintsus

Neurol Neuroimmunol Neuroinflamm is an official journal of the American Academy of Neurology.

Published since April 2014, it is an open-access, online-only, continuous publication journal. Copyright $(\subset$ 2016 American Academy of Neurology. All rights reserved. Online ISSN: 2332-7812.

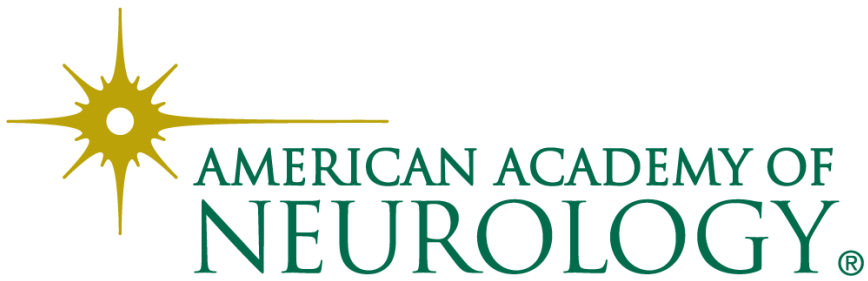

